\title{
Paraunitary Oversampled Filter Bank Design for Channel Coding
}

\author{
Stephan Weiss, ${ }^{1}$ Soydan Redif, ${ }^{1}$ Tom Cooper, ${ }^{2}$ Chunguang Liu, ${ }^{1}$ Paul D. Baxter, ${ }^{2}$ and John G. McWhirter ${ }^{2}$ \\ ${ }^{1}$ Communications Research Group, School of Electronics and Computer Science, University of Southampton, \\ Southampton SO17 1BJ, UK \\ ${ }^{2}$ Advanced Signal and Information Processing Group, QinetiQ Ltd, Malvern, Worcestershire WR14 3PS, UK
}

Received 20 September 2004; Revised 25 July 2005; Accepted 26 July 2005

\begin{abstract}
Oversampled filter banks (OSFBs) have been considered for channel coding, since their redundancy can be utilised to permit the detection and correction of channel errors. In this paper, we propose an OSFB-based channel coder for a correlated additive Gaussian noise channel, of which the noise covariance matrix is assumed to be known. Based on a suitable factorisation of this matrix, we develop a design for the decoder's synthesis filter bank in order to minimise the noise power in the decoded signal, subject to admitting perfect reconstruction through paraunitarity of the filter bank. We demonstrate that this approach can lead to a significant reduction of the noise interference by exploiting both the correlation of the channel and the redundancy of the filter banks. Simulation results providing some insight into these mechanisms are provided.
\end{abstract}

Copyright @ 2006 Hindawi Publishing Corporation. All rights reserved.

\section{INTRODUCTION}

The redundancy and design freedom afforded by oversampled filter banks (OSFBs) has in the past been exploited for robustness towards quantisation of subband signals [1-3], reconstruction of erased or erroneously received subband samples $[4,5]$, or for the design of error correction coders $[6,7]$. More recently, in [8], a systematic parallelism between block codes and oversampled filter bank systems for channel coding has been drawn, whereby the system design is based on unquantised "soft-input" signals [9].

The channel coding schemes in $[2,3,6-9]$ are based on an encoding stage using a preset analysis filter bank. The design freedom afforded in the decoding stage formed by the oversampled synthesis filter bank is then utilised to find the solution that reconstructs the signal-either perfectly or in the mean-square error sense-while ideally projecting away from the noise. The filter banks in [6-9] are constructed from FFTs, which leads to low-cost implementations, and have been shown to be very robust towards burst-type errors, and are easily compatible with OFDM-based modulation system.

If the additive channel noise is correlated, the projection in [8] is performed in the direction of the principal components of the noise subspace, which ideally is restricted such that a noise-free signal subspace exists. Also, in [6-9], the synthesis design is, despite some degrees of freedom (DOFs) due to oversampling, limited by the a priori choice of the analysis filter bank. In [10], the synthesis filter bank is given more flexibility by the design aiming at the suppression of the channel noise under the constraint of invertibility, such that an analysis filter bank encoder can be derived from the synthesis bank. However, the filter bank design in [10] is based on a crude iterative method that can prove the potential of the approach but is otherwise far from being optimal.

Therefore, in this paper, we follow the channel coding scheme in [10] for a correlated additive Gaussian noise channel, but apply a considerably improved constrained synthesis filter bank design method based on the second-order sequential best rotation (SBR2) algorithm [11]. By linking the remaining noise variance after decoding to the covariance matrix of the channel noise as a function of the synthesis filter bank, a suitable broadband eigenvalue decomposition using SBR2 leads to a paraunitary filter bank design that exploits both the correlation of the channel noise as well as the DOFs provided by the OSFBs.

The paper is organised as follows. Based on a brief description of filter banks in Section 2, the general channel coding structure is presented. With the aim of minimising the impact of additive channel noise on the decoded signal, we derive a noise power term, which can be utilised as a cost function for the channel coder design. The proposed constrained optimisation scheme for the synthesis filter bank is outlined in Section 3, which aims to minimise the channel noise power at the decoder output subject to the 


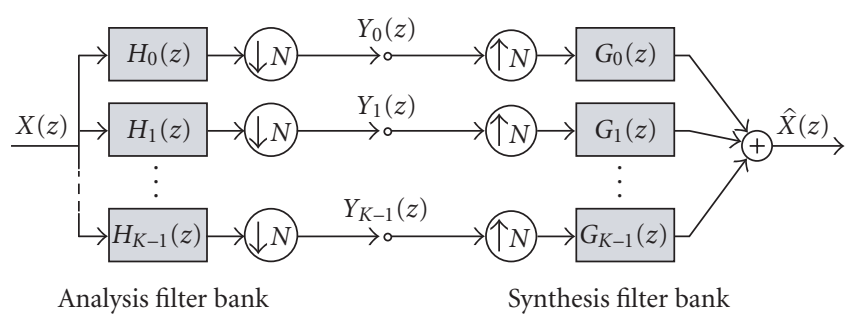

Figure 1: Subband decomposition of a signal $X(z)$.

filter bank being paraunitary, and therefore perfectly reconstructing. Some insight into the functioning of the channel coder design is provided by simulation in Section 4. Conclusions are drawn in Section 5.

In terms of notation, vector quantities are denoted by either lowercase boldface or underscored variables, such as $\mathbf{v}$ or $\underline{V}$, while matrix quantities are boldface uppercase, such as $\mathbf{R}$. Indexed vectors or matrices refer to quantities with polynomial entries, such as $\mathbf{H}(z)$. Finally, a transform pair, such as the Fourier or $z$-transform, is denoted as $h[n] \circ \longrightarrow H\left(e^{j \Omega}\right)$ or $h[n] \circ \longrightarrow H(z)$, respectively.

\section{SYSTEM MODEL}

Based on the description of basic filter bank structures in Section 2.1 and their polyphase description in Section 2.2, a model of the proposed encoder and decoder together with the transmission model is discussed in Section 2.3.

\subsection{Oversampled filter banks}

Figure 1 shows a general filter bank structure comprising of an analysis and a synthesis stage. The analysis filter bank splits a full-band signal $X(z)$ into $K$ frequency bands by a series of bandpass filters $H_{k}(z), k=0,1, \ldots, K-1$, and decimates by a factor $N \leq K$, resulting in so-called "subband" signals $Y_{k}(z)$. The dual operation of reconstructing a fullband signal from the $K$ subband signals is accomplished by a synthesis filter bank, where upsampling by $N$ is followed by interpolation filters $G_{k}(z), k=0,1, \ldots, K-1$.

The purpose of oversampling by a ratio $K / N>1$ rather than a critical decimation by $K$ has application-specific reasons, and has in the past, for example, enabled subband adaptive filtering techniques for acoustic echo cancellation [12], beamforming [13-15], or equalisation [16] by permitting independent processing of the subband signals. In these cases, the filters have to be highly frequency selective, and the redundancy introduced through oversampling is located in the spectral overlap region of the filters within the filter bank system.

The redundancy afforded by OSFBs has more recently attracted attention for channel coding $[6,7]$. There, a code rate $N / K<1$ can ensure robustness against noise interference, with the aim of restoring noise-corrupted samples due to the redundant format in which the data is transmitted. The analysis and synthesis filter banks function as encoder and decoder, while the filters $H_{k}(z)$ and $G_{k}(z)$ are no longer limited to a bandpass design, but will rather be selected according to the characteristics of the interfering noise.

\subsection{Polyphase matrices}

For implementation and analysis purposes, OSFBs as shown in Figure 1 are conveniently represented by polyphase analysis and synthesis matrices. The former is based on a type-I polyphase expansion of the analysis filters $H_{k}(z)$ [17]:

$$
H_{k}(z)=\sum_{n=0}^{N-1} z^{-n} H_{k, n}\left(z^{N}\right),
$$

with polyphase components $H_{k, n}(z)$, and a type-II decomposition [17] of the input signal

$$
X(z)=\sum_{n=0}^{N-1} z^{-N+n-1} X_{n}\left(z^{N}\right),
$$

with polyphase components $X_{n}(z)$. This allows us to denote the vector of subband signals $\underline{Y}(z)$ as

$$
\underbrace{\left[\begin{array}{c}
Y_{0}(z) \\
\vdots \\
Y_{K-1}(z)
\end{array}\right]}_{\underline{Y}(z)}=\underbrace{\left[\begin{array}{ccc}
H_{0,0}(z) & \cdots & H_{0, N-1}(z) \\
\vdots & \ddots & \vdots \\
H_{K-1,0}(z) & \cdots & H_{K-1, N-1}(z)
\end{array}\right]}_{\mathbf{H}(z)} \underbrace{\left[\begin{array}{c}
X_{0}(z) \\
\vdots \\
X_{N-1}(z)
\end{array}\right]}_{\underline{X}(z)} .
$$

Therefore, the filter bank can be represented by a demultiplexing of the input signal into $N$ lines, followed by a multiple-input multiple-output (MIMO) system described by the polyphase analysis matrix $\mathbf{H}(z)$. This structure is seen as part of Figure 2 .

Analogously, a polyphase synthesis matrix $\mathbf{G}(z) \in$ $\mathbb{C}^{N \times K}(z)$ can be defined based on a polyphase expansion of $G_{k}(z)$, yielding the synthesis filter bank representation in Figure 2 comprising $\mathbf{G}(z)$ followed by an $N$-fold multiplexer.

A filter bank system is perfectly reconstructing if

$$
\mathbf{G}(z) \mathbf{H}(z)=z^{-\Delta} \mathbf{I}_{N} .
$$

The design of such a system can be demanding in terms of the number of coefficients that need to be optimised. A reduction of the parameter space by, for example, deriving all $K$ filters from a prototype by modulation $[2,18]$ or by permitting only symmetric filter impulse responses $[4,18]$ often makes the problem tractable. 


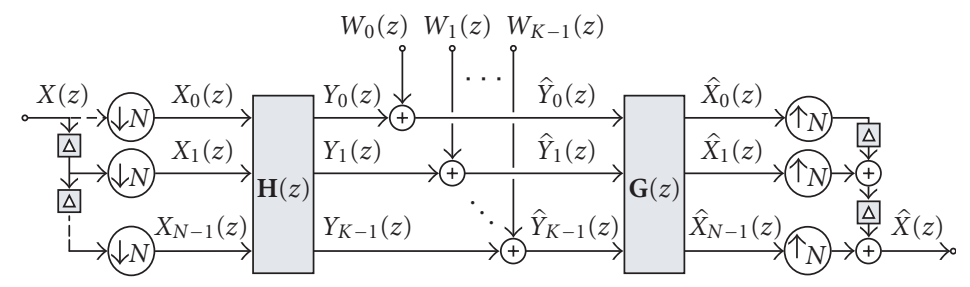

FIGURE 2: General setup of a channel coder based on $K$ channel analyses and synthesis filter banks, arranged around the transmission over $K$ additive Gaussian noise channels.

\subsection{Setup and channel coder}

The overall model of the considered system is provided in Figure 2. In the transmitter, the $N$ polyphase components of $X(z)$ are encoded by the polyphase analysis matrix $\mathbf{H}(z)$. The transmission could either employ $K$ separate channels as shown in Figure 2, or multiplex the $K$ encoder outputs onto a single signal transmitted over a single-input singleoutput channel. This channel is subject to corruption by additive Gaussian wide-sense-stationary (WSS) noise, and for simplicity is assumed to be nondispersive.

In the case of a dispersive channel, the model in Figure 2 can also be applied, if an ideal zero-forcing (ZF) equaliser is employed prior to decoding by the polyphase synthesis matrix $\mathbf{G}(z)$. While the channel and the ZF equaliser annihilate each other for the signal path, in the noise path, the ZF equaliser can be absorbed into the innovations filter model producing the additive noise components $W_{k}(z)$, $k=0,1, \ldots, K-1$. This absorption would result in an additional shaping of the channel noise corrupting the $K$ received signals $\hat{Y}_{k}(z)$, and provide an additional incentive for channel coding that can exploit the spatiotemporal structure of the noise.

In the receiver after decoding, the polyphase components $\hat{X}_{n}(z)$ can be collected similar to $\underline{X}(z)$ in $(3)$ in a vector $\underline{\hat{X}}(z)$, which is given by

$$
\underline{\hat{X}}(z)=\mathbf{G}(z)(\underline{Y}(z)+\underline{W}(z)),
$$

whereby $\underline{Y}(z)=\mathbf{H}(z) \underline{X}(z) \in \mathbb{C}^{K}(z)$ and $\underline{W}(z) \in \mathbb{C}^{K}(z)$ contain the subband signal components of the transmitted data and the noise, respectively. Selecting perfect reconstruction filter banks $\mathbf{G}(z) \mathbf{H}(z)=\mathbf{I}_{N}$,

$$
\underline{E}(z)=\underline{X}(z)-\underline{\hat{X}}(z)=-\mathbf{G}(z) \underline{W}(z)
$$

is obtained.

In order to assess the total received noise variance $\sigma_{e}^{2}$ in $\hat{X}(z)$, let the $N$-element vector $\mathbf{e}[m]$ contain the $N$ time series associated with the $z$-domain quantities in $\underline{E}(z) \bullet-\mathbf{e}[m]$, which depend on the time index $m$ in the decimated domain. Thus, we have

$$
\sigma_{e}^{2}=\frac{1}{N} \operatorname{tr}\left\{\mathscr{E}\left\{\mathbf{e}[m] \mathbf{e}^{\mathrm{H}}[m]\right\}\right\},
$$

where $\operatorname{tr}\{\cdot\}$ denotes trace and $\mathscr{E}\{\cdot\}$ is the expectation operator. Defining the autocorrelation matrix

$$
\mathbf{R}_{e e}[\tau]=\mathcal{E}\left\{\mathbf{e}[m] \mathbf{e}^{\mathrm{H}}[m-\tau]\right\}
$$

and its $z$-transform $\mathbf{R}_{e e}(z) \bullet \longrightarrow \mathbf{R}_{e e}[\tau]$ denoting the power spectrum of the process $\mathbf{e}[m][17]$, the noise variance is given by

$$
\begin{aligned}
\sigma_{e}^{2} & =\frac{1}{N} \operatorname{tr}\left\{\mathbf{R}_{e e}[0]\right\}=\left.\frac{1}{N} \operatorname{tr}\left\{\mathbf{R}_{e e}(z)\right\}\right|_{z=0} \\
& =\left.\frac{1}{N} \operatorname{tr}\left\{\mathbf{G}(z) \mathbf{R}_{w w}(z) \tilde{\mathbf{G}}(z)\right\}\right|_{z=0} .
\end{aligned}
$$

The notation in (10) uses the para-Hermitian operator $\widetilde{\{\cdot\}}$, which applies a complex conjugate transposition and a time reversal [17] to its operand. Note that (6) has been exploited to trace the noise variance back to the power spectrum $\mathbf{R}_{w w}(z)$, which is the $z$-transform of the covariance matrix of the channel noise,

$$
\mathbf{R}_{w w}[\tau]=\mathcal{E}\left\{\mathbf{w}[m] \mathbf{w}^{\mathrm{H}}[m-\tau]\right\},
$$

with $\mathbf{w}[m] \circ \longrightarrow \underline{W}(z)$ as defined in Figure 2.

\section{CHANNEL CODER AND FILTER BANK DESIGN}

Based on the idea of the channel coder outlined in Section 3.1 , this section considers a suitable factorisation of the power spectrum at the decoder output in Section 3.2, admitting a useful coder design in Section 3.3. An algorithm to construct filter banks achieving this design is reviewed in Section 3.4.

\subsection{Proposed coding approach}

It is the quantity $\sigma_{e}^{2}$ in (7) which is generally minimised in some sense in channel coding. In [8], for a given $\mathbf{H}(z)$, the degrees of freedom (DOFs) in the design of $\mathbf{G}(z)$ are exploited to minimise $\sigma_{e}^{2}$ in the MSE sense. Note however that this approach limits the DOFs that can be dedicated to fit the synthesis matrix to the spatiotemporal structure of the noise.

Therefore, we proposed to minimise (7) by optimising $\mathbf{G}(z)$ without restriction by a specific $\mathbf{H}(z)$. The only condition placed on $\mathbf{G}(z)$ is that it admits a right inverse $\mathbf{G}^{\dagger}(z)$ such that $\mathbf{G}(z) \mathbf{G}^{\dagger}(z)=z^{-\Delta}$. A stronger restriction than simple invertibility placed on $\mathbf{G}(z)$ is paraunitarity, which however has two important advantages: (i) the analysis filter bank is immediately given by $\mathbf{H}(z)=\widetilde{\mathbf{G}}(z)$, and (ii) paraunitarity provides a minimum-norm solution such that the transmit power is limited. As a counterexample, an invertible $\mathbf{G}(z)$ might elicit an ill-conditioned $\mathbf{H}(z)$ which may attempt to 
transmit highly powered signals over subspaces associated with near-rank deficiency.

\subsection{Factorisation of the noise covariance matrix}

We approach the minimisation of (10) via a factorisation of the power spectrum

$$
\mathbf{R}_{w w}(z)=\mathbf{U}(z) \boldsymbol{\Gamma}(z) \tilde{\mathbf{U}}(z)
$$

such that $\mathbf{U}(z) \in \mathbb{C}^{K \times K}(z)$ is paraunitary and strongly decorrelates $\mathbf{R}_{w w}(z)$, that is,

$$
\Gamma(z)=\operatorname{diag}\left\{\Gamma_{0}(z), \Gamma_{1}(z), \ldots, \Gamma_{K-1}(z)\right\}
$$

is a diagonal matrix with polynomial entries $\Gamma_{k}(z)$. This factorisation presents a broadband eigenvalue decomposition, which can be further specified by demanding $\Gamma(z)$ to be spectrally majorised $[11,19]$ such that the power spectral density (PSD) of the $k$ th noise component $\Gamma_{k}\left(e^{j \Omega}\right)=\left.\Gamma_{k}(z)\right|_{z=e^{j \Omega}}$ evaluated on the unit circle obeys

$$
\Gamma_{k}\left(e^{j \Omega}\right) \geq \Gamma_{k+1}\left(e^{j \Omega}\right) \quad \forall \Omega, k=0,1, \ldots, K-2,
$$

similar to the ordering of the singular values in a singular value decomposition. Note that paraunitarity or losslessness of $\mathbf{U}(z)$ conserves power, that is, $\left.\operatorname{tr}\{\boldsymbol{\Gamma}(z)\}\right|_{z=0}=$ $\left.\operatorname{tr}\left\{\mathbf{R}_{w w}(z)\right\}\right|_{z=0}$.

\subsection{Channel coding design}

Using the redundancy $N<K$ due to oversampling, we can construct $\mathbf{G}(z)$ from $\mathbf{U}(z)$ to select the lower (and therefore smallest) $N$ elements on the main diagonal of $\boldsymbol{\Gamma}(z)$. Let

$$
\mathbf{U}(z)=\left[\underline{U}_{0}(z) \underline{U}_{1}(z) \cdots \underline{U}_{K-1}(z)\right],
$$

then

$$
\mathbf{G}(z)=\left[\begin{array}{c}
\underline{\tilde{U}}_{K-N}(z) \\
\underline{\tilde{U}}_{K-N+1}(z) \\
\vdots \\
\underline{\tilde{U}}_{K-1}(z)
\end{array}\right] \in \mathbb{C}^{N \times K}(z),
$$

such that $\mathbf{G}(z) \mathbf{U}(z)=\left[\mathbf{0}_{N \times K-N} \mathbf{I}_{N}\right]$. If

$$
\boldsymbol{\Gamma}(z)=\left[\begin{array}{ll}
\boldsymbol{\Gamma}_{00}(z) & \boldsymbol{\Gamma}_{01}(z) \\
\boldsymbol{\Gamma}_{10}(z) & \boldsymbol{\Gamma}_{11}(z)
\end{array}\right]
$$

with $\Gamma_{11}(z) \in \mathbb{C}^{N \times N}$ and the remaining submatrices of appropriate dimension, then the noise power at the decoder output becomes

$$
\begin{aligned}
\sigma_{e}^{2} & =\left.\frac{1}{N} \operatorname{tr}\left\{\boldsymbol{\Gamma}_{11}(z)\right\}\right|_{z=0} \\
& =\frac{1}{N} \sum_{i=K-N}^{K-1} \int_{0}^{2 \pi} \Gamma_{i}\left(e^{j \Omega}\right) d \Omega .
\end{aligned}
$$

Therefore, the spectral majorisation in the broadband eigenvalue decomposition (12) is essential to the success of the proposed channel coder design.

\subsection{Sequential best rotation algorithm}

In order to achieve the factorisation in (12) fulfilling spectral majorisation according to (14), we use the second-order sequential best rotation (SBR2) algorithm [11]. In the following, only a brief description of the algorithm is provided, while for an in-depth treatment, the reader is referred to $[11,20]$.

SBR2 is an iterative broadband eigenvalue decomposition technique based on second-order statistics only and can be seen as a generalisation of the Jacobi algorithm. The decomposition after $L$ iterations is based on a paraunitary matrix

$$
\mathbf{U}_{L}(z)=\prod_{i=0}^{L} \mathbf{Q}_{i} \boldsymbol{\Lambda}_{i}(z),
$$

whereby $\mathbf{Q}_{i}$ is a Givens rotation and the matrix $\boldsymbol{\Lambda}_{i}(z)$ is a paraunitary matrix of the form

$$
\Lambda_{i}(z)=\mathbf{I}-\mathbf{v}_{i} \mathbf{v}_{i}^{\mathrm{H}}+z^{-\Delta_{i}} \mathbf{v}_{i} \mathbf{v}_{i}^{\mathrm{H}},
$$

with $\mathbf{v}_{i}=\left[\begin{array}{lllllll}0 & \cdots & 0 & 1 & 0 & \cdots & 0\end{array}\right]^{\mathrm{H}}$ containing zeros except for a unit element in the $\delta_{i}$ th position. Thus, $\boldsymbol{\Lambda}_{i}(z)$ is an identity matrix with the $\delta_{i}$ th diagonal element replaced by a delay $z^{-\Delta_{i}}$.

At the ith step, SBR2 will eliminate the largest offdiagonal element of the matrix $\mathbf{U}_{i-1}(z) \mathbf{R}_{w w}(z) \widetilde{\mathbf{U}}_{i-1}(z)$, which is defined by the two corresponding subchannels and by a specific lag index. By delaying the two contributing subchannels appropriately with respect to each other by selecting the position $\delta_{i}$ and the delay $\Delta_{i}$, the lag value is compensated. Thereafter, a Givens rotation $\mathbf{Q}_{i}$ can eliminate the targeted element such that the resulting two terms on the main diagonal are ordered in size, leading to a diagonalisation and at the same time accomplishing a spectral majorisation.

Hence, each step comprises of optimising the parameter set $\left\{\delta_{i}, \Delta_{i}, \theta_{i}\right\}$. While the largest off-diagonal element in $\mathbf{U}_{i-1}(z) \mathbf{R}_{w w}(z) \tilde{\mathbf{U}}_{i-1}(z)$ is eliminated, the remainder of the matrix is also affected. In extensive simulations, SBR2 has proven very robust and stable in achieving both a diagonalisation and spectral majorisation of any given covariance matrix, whereby the algorithm is stopped either after reaching a certain measure for suppressing off-diagonal terms or after exceeding a defined number of iterations $[11,20]$. The order $O_{\text {OSFB }}$ of the filter bank defined by the paraunitary polyphase matrix $\mathbf{U}_{L}(z)$ is bounded by $O_{\mathrm{OSFB}} \leq \sum_{i=0}^{L} \Delta_{i}$. Since the individual delays $\Delta_{i}$ are optimised by the algorithm and not known a priori, the filter bank order $O_{\mathrm{OSFB}}$ cannot be determined or limited a priori to applying SBR2 to the power spectral matrix $\mathbf{R}_{w w}(z)$.

\section{SIMULATIONS AND RESULTS}

To illustrate the proposed channel coding design, three design examples are demonstrated in the following. The first design assumes an independent transmission across $K$ subchannels, while the latter two are based on a timemultiplexed transmission leading to correlation between the $K$ virtual subchannels. 


\subsection{Multichannel transmission}

We assume the transmission scenario shown in Figure 2, whereby $K$ subchannels are available and are corrupted by Gaussian noise processes $w_{k}[m], k=0,1, \ldots, K-1$, such that

$$
\mathcal{E}\left\{w_{k}[m] w_{j}[m-\tau]\right\}= \begin{cases}0 & \text { for } k \neq j, \\ r_{k}[\tau] \circ \longrightarrow R_{k}\left(e^{j \Omega}\right) & \text { for } k=j .\end{cases}
$$

Specifically, for the example below, we assume that $K=6$ and that the $w_{k}[m]$ are produced by uncorrelated unit variance and zero-mean Gaussian processes by passing through innovation filters $p_{k}[m] \circ \longrightarrow P_{k}(z)[21]$,

$$
\begin{gathered}
{\left[\begin{array}{l}
P_{0}(z) \\
P_{1}(z) \\
P_{2}(z) \\
P_{3}(z)
\end{array}\right]=\left[\begin{array}{cccc}
1 & 1 & 1 & 1 \\
1 & -1 & 1 & -1 \\
1 & 1 & -1 & -1 \\
1 & -1 & -1 & 1
\end{array}\right] \cdot\left[\begin{array}{c}
1 \\
z^{-1} \\
z^{-2} \\
z^{-3}
\end{array}\right],} \\
P_{4}(z)=P_{5}(z)=10,
\end{gathered}
$$

such that $r_{k}[\tau]=\sum_{m} p_{k}[m] p_{k}^{*}[m-\tau]$. The resulting power spectrum $\mathbf{R}_{w w}(z)$ is a diagonal matrix with $\operatorname{PSDs} R_{k}\left(e^{j \Omega}\right)$ as defined in (21) and shown in Figure 3(a) on its diagonal.

Prior to running the SBR2 algorithm on $\mathbf{R}_{w w}(z)$, its purely diagonal structure must be perturbed through the application of an arbitrary paraunitary matrix. Thereafter, independent of this perturbation, SBR2 achieves a diagonalisation of $\Gamma(z)$ after $L \approx 250$ iterations, whereby a ratio of approximately $10^{-3}$ between the energy of off-diagonal and on-diagonal terms is reached. However, recall from (17)(18) that the minimisation of the noise power $\sigma_{e}^{2}$ at the decoder output does not necessitate the diagonality of $\Gamma(z)$ but does strongly depend on its spectral majorisation. To examine the latter after convergence of SBR2, the PSDs of the main diagonal elements $\Gamma_{k}\left(e^{j \Omega}\right)$ are depicted in Figure 3(b). Quite clearly, except for a low-power region of the bands $\Gamma_{4}\left(e^{j \Omega}\right)$ and $\Gamma_{5}\left(e^{j \Omega}\right)$ near $\Omega=\pi$, spectral majorisation has been achieved in the sense of (14). Interestingly, the general shape of the PSDs in Figure 3(b) closely follows those in Figure 3(a), but frequency intervals have been reassigned to different subchannels and have been ordered in descending power.

Integrating over the PSDs in Figure 3 provides the noise variance of the various subchannels, which are illustrated in Figure 4 for $\mathbf{R}_{w w}(z)$ and $\boldsymbol{\Gamma}(z)$ without and with coding, respectively. The coder would then utilise those $N$ coded subchannels represented in $\Gamma(z)$ that carry the lowest noise power. These $N$ coded subchannels convey the $N$ polyphase components of the transmitted signal $X(z)$, which according to Figure 4 are subject to different levels of noise. Note that the polyphase component transmitted over the lowest subchannel provides the best protection against noise, while noise introduced on higher subchannels increases in power. This fact can be exploited for unequal error protection to, for example, high-quality high-speed video transmission.

In order to demonstrate how the residual noise power in the decoded subchannels depends on the order of the filter

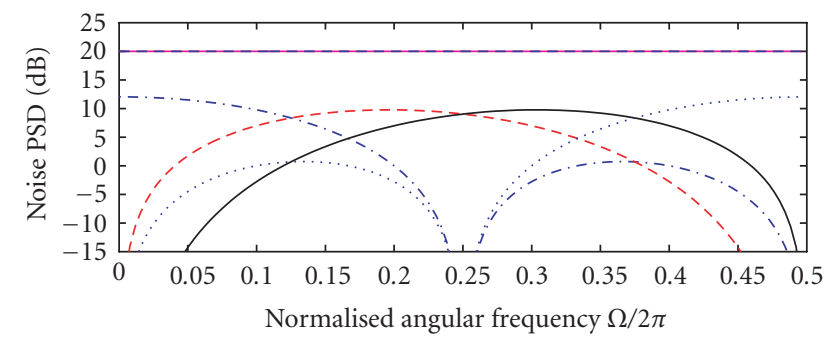

$$
\begin{array}{ll}
\cdots-R_{0}\left(e^{j \Omega}\right) & \cdots \cdots R_{3}\left(e^{j \Omega}\right) \\
---R_{1}\left(e^{j \Omega}\right) & -R_{4}\left(e^{j \Omega}\right) \\
-R_{2}\left(e^{j \Omega}\right) & ---R_{5}\left(e^{j \Omega}\right)
\end{array}
$$

(a)

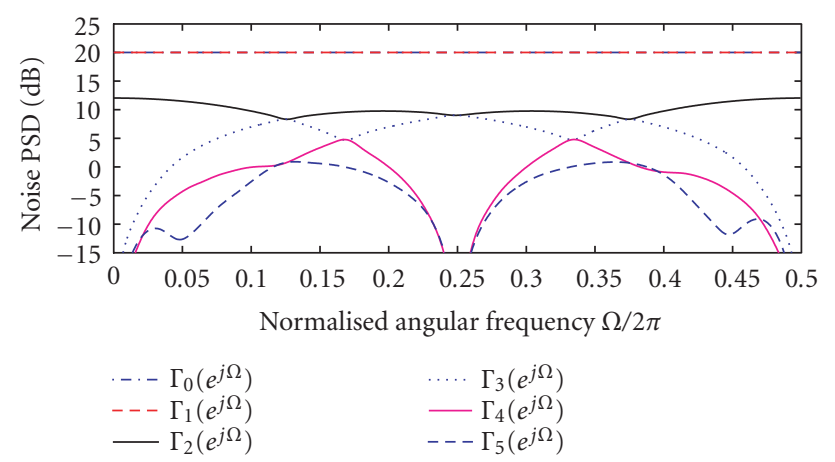

(b)

FIGURE 3: PSDs on the main diagonals of (a) the power spectrum $\mathbf{R}_{w w}(z)$ of the channel noise consisting of the $R_{k}\left(e^{j \Omega}\right)$ of $(21)$ and (b) $\boldsymbol{\Gamma}(z)$ after application of the SBR2 algorithm.

bank, Figure 5(a) provides an evolution of the total received noise power in dependence on the number of iterations used for SBR2 and on the number of selected subchannels $N$. If all subchannels are selected, that is, $N=K=6$, no redundancy can be exploited and the total noise power cannot be reduced. For $N<4$, the channel characteristics permit the exploitation of low-noise subspaces, which is achieved through spectral majorisation of the power spectrum due to the filter banks. Note that in Figure 5, initially a small degradation of the cumulative noise powers for $N<6$ with respect to (21) occurs as a result of the random perturbance of the diagonal $\mathbf{R}_{w w}(z)$ by an arbitrary paraunitary matrix. It is evident from Figure 5 that the required filter order, and therefore the complexity of the resulting filter bank, depends on the code rate, that is, the lower $N$ and hence the higher the oversampling ratio, the more iterations are required to fully exploit the available potential in reducing the output noise power $\sigma_{e}^{2}=\sum_{k=K-N}^{K-1} \gamma_{k}[0]$. The order of the polynomial matrix $\mathbf{U}_{L}(z)$, and therefore the filter bank matrices $\mathbf{H}(z)$ and $\mathbf{G}(z)$ after $L$ iterations, is given in Figure 5(b), whereby tails of the filters can be truncated if a lower numerical resolution is sufficient. In the case of channel coding, infinite numerical precision would be wasteful, while quantisation noise is acceptable if its power is well below the level of residual channel noise in Figure 5(a). 


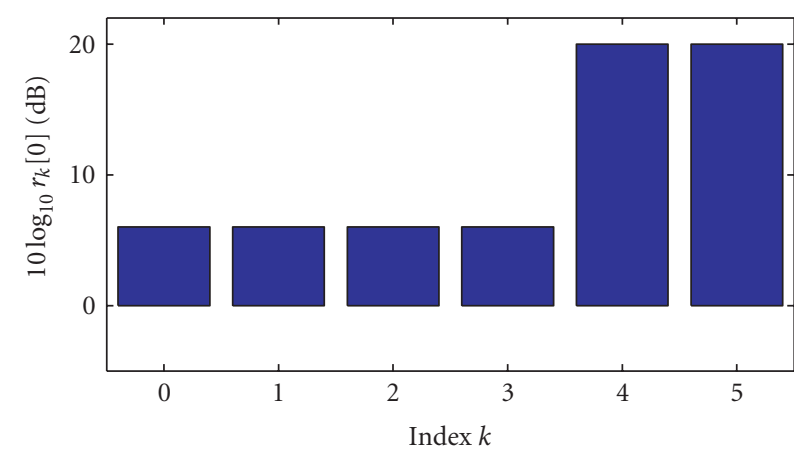

(a)

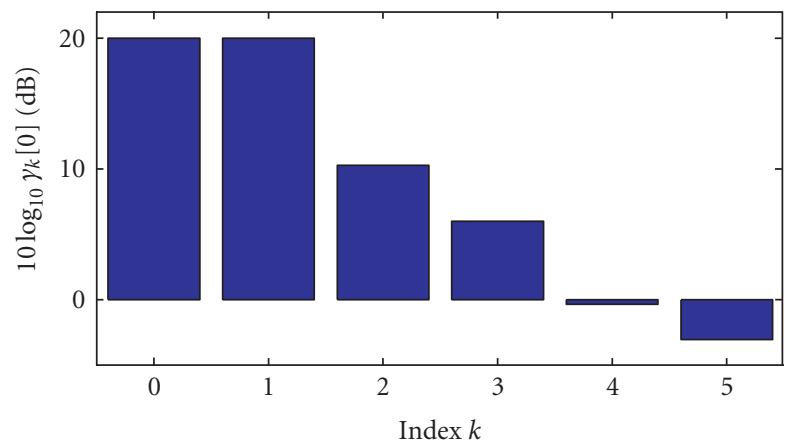

(b)

Figure 4: Variances of (a) uncoded noise $r_{k}[0]$ and (b) coded subchannels $\gamma_{k}[0]=(1 / 2 \pi) \int_{0}^{2 \pi} \Gamma_{k}\left(e^{j \Omega}\right) d \Omega$.

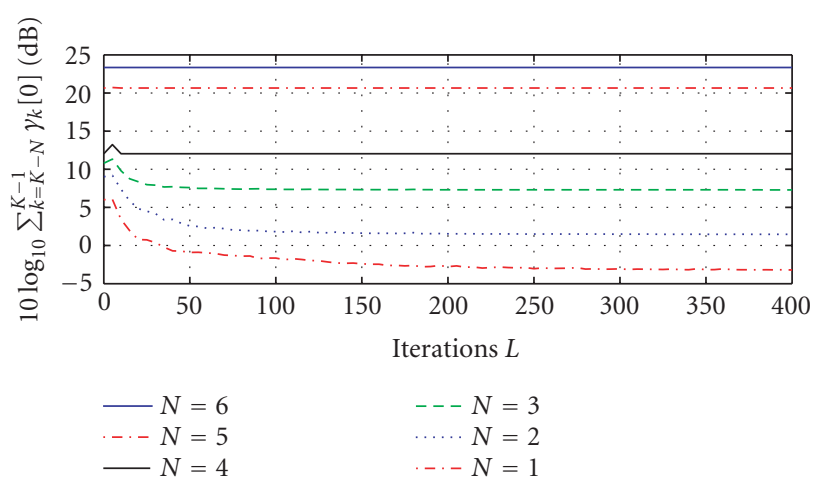

(a)

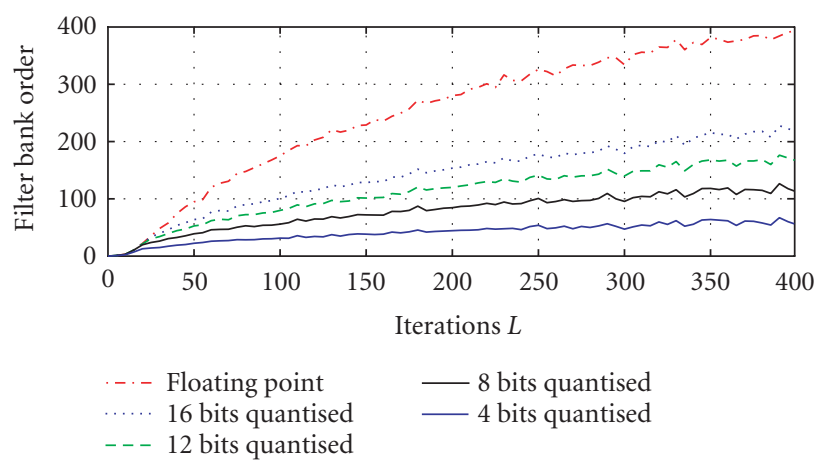

(b)

FIGURE 5: (a) cumulative variance of $N$ subchannels containing the lowest noise power after $L$ iterations of SBR2 and (b) filter bank order after $L$ iterations; the curves are averaged over 50 random trials with different paraunitary matrices perturbing the originally diagonal $\mathbf{R}_{w w}(z) ; \gamma_{k}[0]$ is defined in Figure 4.

If a decimation factor of $N=2$ is chosen for the filter banks, only the two coded subchannels with the lowest noise variance in Figure 4(b) will be utilised. The reduction in noise power results in an SNR enhancement of the coded scheme with respect to a transmission scenario of identical symbol throughput based on maximum-ratio combining of the $K=6$ channels in Figure $4(\mathrm{a})$ of $7.5 \mathrm{~dB}$. Note that a maximum-ratio combiner uses a zero-order diagonal $\mathbf{G}(z)$, and accordingly $\mathbf{H}(z)$ with the elements inversely proportional to the standard deviation of the noise in the subchannels.

Some insight into how the reduction of noise power is gained by the proposed coding method for the case $N=2$ is demonstrated in Figure 6, where the resulting characteristics of a $K=6$ channel filter banks decimated by $N=2$ are shown. The displayed characteristics refer to the filter bank structure given in Figure 1, and are plotted against the PSDs of the channel noise after $N=2$-fold expansion. Figure 6 very clearly underlines the functioning of the coder, which effectively excludes the two subchannels with high noise power from transmission, while in all other subchannels, the transmitted power is concentrated in frequency bands where the noise PSD assumes its lowest values.

\subsection{Time-multiplexed transmission}

In the following, we consider the case where the noise in the $K$ subchannels in Figure 2 may be mutually correlated. This can occur through a time-multiplexed transmission of the $K$ encoded symbols over the same channel corrupted by noise $w[m]$, which is assumed to be modelled as a unit-variance zero-mean Gaussian WSS process undergoing an innovation filter $p[m]$. Therefore, the autocorrelation function of $w[m]$ is given by $r[\tau]=\sum_{m} p[m] p^{*}[m-\tau] \circ \longrightarrow R(z)$. After demultiplexing into $K$ channels in the receiver, the resulting noise power spectrum $\mathbf{R}_{w w}(z)$ can be shown to be given by the pseudocirculant matrix

$$
\mathbf{R}_{w w}(z)=\left[\begin{array}{cccc}
R_{0}(z) & R_{1}(z) & \cdots & R_{K-1}(z) \\
z^{-1} R_{K-1}(z) & R_{0}(z) & & R_{K-2}(z) \\
\vdots & \ddots & \ddots & \vdots \\
z^{-1} R_{1}(z) & \cdots & z^{-1} R_{K-1}(z) & R_{0}(z)
\end{array}\right]
$$

containing the $K$ polyphase components $R_{k}(z), k=0,1, \ldots$, $K-1$, of $R(z)$,

$$
R(z)=\sum_{k=0}^{K-1} R_{k}\left(z^{K}\right) z^{-k}
$$



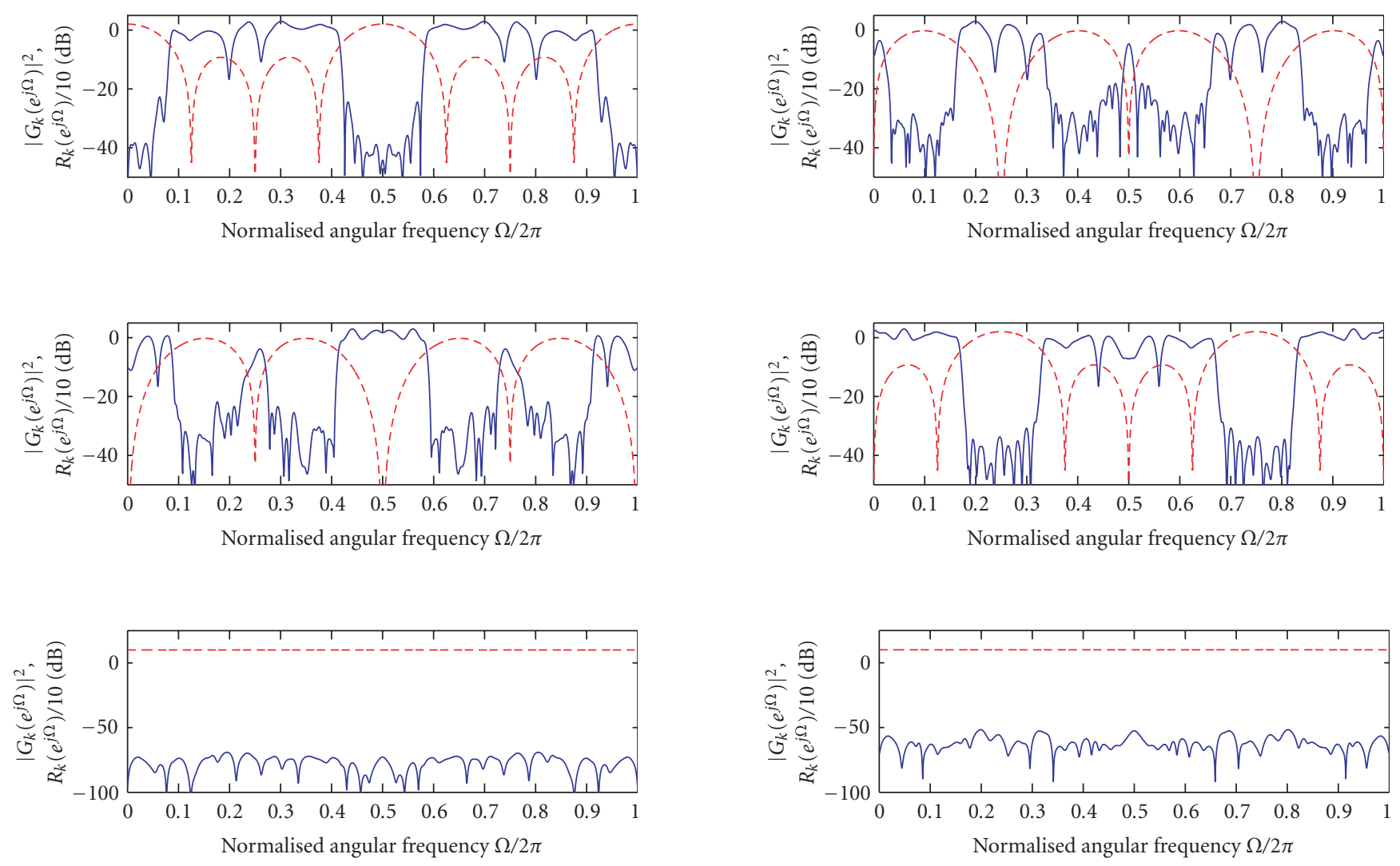

FIGURE 6: PSDs of channel noise processes $w_{k}[m], k=0,1, \ldots, K-1$, decimated by $N=2$ (dashed) and magnitude responses of the filters $\left|G_{k}\left(e^{j \Omega}\right)\right|=\left|H_{k}\left(e^{j \Omega}\right)\right|$ (solid).

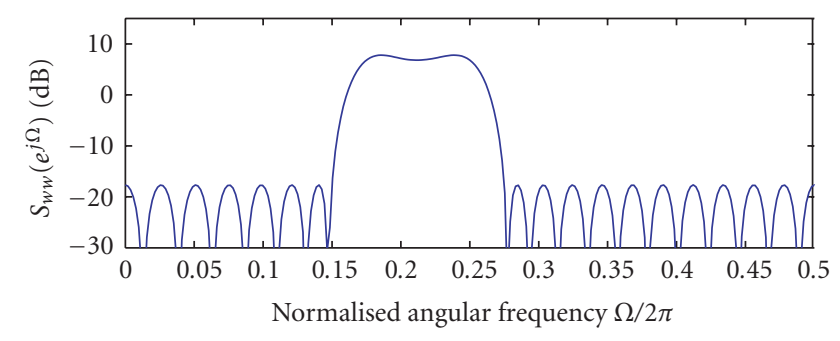

FIgURE 7: Channel noise PSD in time multiplex Channel II.

\section{Channell}

In a first case, the multiplex channel is assumed to be corrupted by an interfering radio signal occupying a quarter of the available bandwidth. The interference is modelled by a zero-mean unit-variance white Gaussian noise exciting a 49th-order bandpass FIR filter, which results in the channel noise PSD shown in Figure 7. The PSD within each of the subchannels described by $\mathbf{R}_{w w}(z)$ for any given $K$ is identical. Here, different from Section 4.1, the coder has to additionally exploit the correlation between the $K$ subchannels. After application of the SBR2 algorithm, the reduction in noise power-the ratio between the output power of the coder to

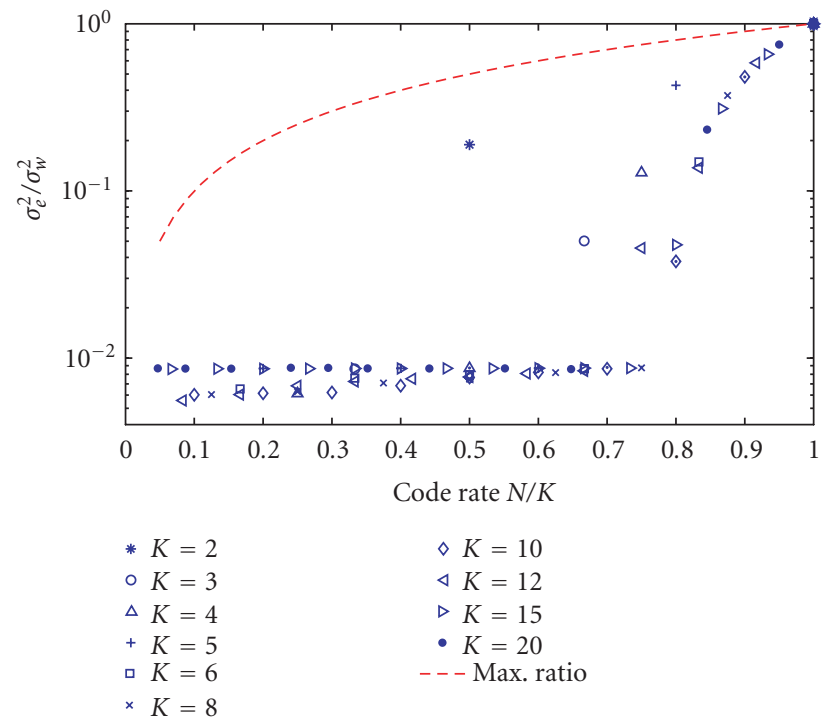

FIGURE 8: Noise reduction achieved by the proposed coding scheme over Channel II characterised in Figure 7.

the power of the channel noise process $w[m]$-for various choices of $K$ and $N$ is depicted in Figure 8. In comparison to 


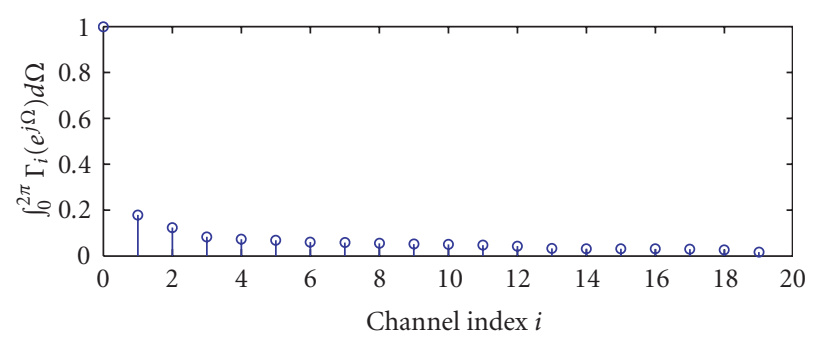

FIGURE 9: Spectral majorisation in the decomposition of the noise power spectral matrix $R(z)$ by SBR2 for $K=20$ channels.

maximum-ratio combining with identical symbol throughput, the proposed coder in general performs consistently and considerably better, whereby an increase in $K$ permits both a finer resolution to exploit spatial correlation as well as the use of more flexible code rates $N / K$.

The proposed channel coder can exploit the spectral characteristics of the channel noise well, and, provided a sufficient resolution of the code rate, exhibits an approximately constant output noise power once the code rate reaches the approximately interference-free relative bandwidth of $75 \%$ available over the channel.

\section{Channel II}

We select a power-line communication channel (PLC), whose PSD in a worst-case scenario can be modelled as [22]

$$
S_{\log }(f)=38.75|f|^{-.72} \mathrm{dBm} / \mathrm{Hz} .
$$

Sampled at $30 \mathrm{MHz}$, an iterative least-squares fit has been employed to derive an FIR innovation filter with 256 coefficients to produce the PSD characterised in (25) [23]. Applying SBR2 to the resulting noise power spectrum $\mathbf{R}(z)$, an example for the resulting spectral majorisation is given in Figure 9 for a decomposition into $K=20$ channels, for which SBR2 yields a 37th-order filter bank matrix $\mathbf{H}(z)$. The latter is reached with a stopping criterion of $10^{3}$ for the ratio between the total power and the power contained in offdiagonal elements in $\mathbf{U}_{L}(z) \mathbf{R}_{w w}(z) \tilde{\mathbf{U}}_{L}(z)$. For this broadband eigenvalue decomposition, a single strong eigenmode of the noise is clearly visible. Therefore, if oversampling is applied and the strongest eigenmodes of the noise subspace can be deselected form transmission, the noise power in the decoded signal in the receiver can be significantly reduced. The coding gain for the PLC simulation model in (25) is given in Figure 10 for various selections of channels $K$, and compared to maximum-ratio combining by repeated transmission of symbols over an otherwise uncoded channel.

Figure 10 suggests that the OSFB approach can provide considerable coding gain at a high code rate close to unity for the case of highly correlated noise. In order to exploit this, $K$ has to be chosen sufficiently large in order to offer a high resolution with respect to possible code rates.

In the following, we consider transmitting quadrature amplitude modulated (QAM) symbols over the OSFB-coded

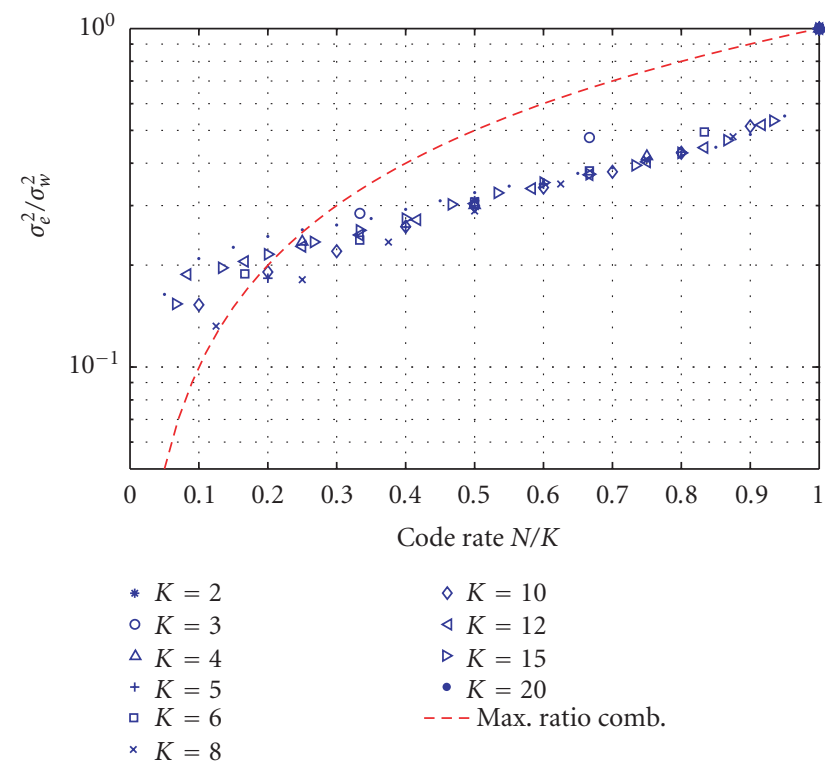

FIgURE 10: Coding gain of the OSFB coder applied to the PLC channel defined in (25) for various values of $K$ and different code rates.

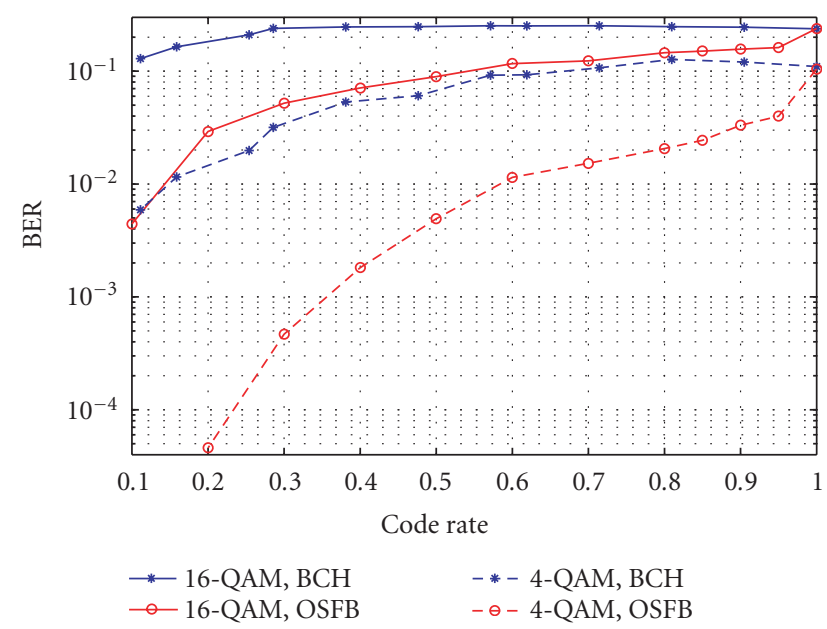

FIGURE 11: BER for coding using $M$-QAM and OSFB and BCH channel coding, in dependency on the code rate.

PLC channel. For a channel SNR of $3 \mathrm{~dB}$, Figure 11 presents results for different code rates for a QPSK/4-QAM and a 16QAM-based transmission.

As a comparison, we also present results for a $\left(63, N_{\mathrm{BCH}}\right)$ $\mathrm{BCH}$-coded PLC channel, where $N_{\mathrm{BCH}}$ is varied to achieve various code rates [23]. The BCH-encoded bit stream is $M$ QAM mapped and transmitted over the PLC channel. In the receiver, after slicing and demapping, a $\mathrm{BCH}$ decoder aims to recover the original bit stream. A $(37,20)$ matrix interleaver, imposing the same processing delay as the OSFB coder, is set to assist in breaking up noise correlation and burst-type errors. Although its computational complexity is higher than the various $\mathrm{BCH}$ coders, it is clear that the OSFB coder provides superior protection against correlated channel noise, 
and almost enables the use of 16-QAM rather than QPSK as opposed to a $\mathrm{BCH}$ coder, thus nearly doubling the data throughput without sacrificing error protection.

\section{CONCLUSIONS}

In this paper, we have proposed a channel coding approach based on OSFBs by first designing a decoder that minimises the influence of correlated channel noise in the receiver, and thereafter obtaining the encoder. By demanding paraunitarity for the decoding OSFB, the latter step is trivial and ensures a strict bound on the transmitted power. An OSFB design method has been proposed, which is based on a broadband eigenvalue decomposition and demonstrates good performance in suppressing the correlated channel noise. Some insight into the effects of the design have been given by considering transmission scenarios over $K$ independent channels or by time-multiplexed transmission, where the exploitation of spatial or spectral correlations can bring substantial benefits over a transmission of identical symbol throughput using maximum-ratio combining of the subchannels.

The SNR enhancement gained from the proposed coding architecture can be utilised in conjunction with the transmission of quantised data such as found in binary-phase shift keying or multilevel QAM symbols, such that the occurrence of symbol or bit errors is reduced. This has been demonstrated by considering a power-line communications scenario, whereby the proposed OSFB design can significantly outperform standard channel coding techniques such as $\mathrm{BCH}$, offering a higher data throughput at identical protection against errors.

\section{ACKNOWLEDGMENT}

We would like to gratefully acknowledge the anonymous reviewers for insightful questions and helpful guidance.

\section{REFERENCES}

[1] Z. Cvetković and M. Vetterli, "Overcomplete expansions and robustness," in Proceedings of IEEE-SP International Symposium on Time-Frequency and Time-Scale Analysis (TFTS '96), pp. 325-328, Paris, France, June 1996.

[2] H. Bölcskei and F. Hlawatsch, "Oversampled filter banks: optimal noise shaping, design freedom, and noise analysis," in Proceedings of IEEE International Conference on Acoustics, Speech, and Signal Processing (ICASSP'97), vol. 3, pp. 2453-2456, Munich, Germany, April 1997.

[3] H. Bölcskei and F. Hlawatsch, "Noise reduction in oversampled filter banks using predictive quantization," IEEE Transactions on Information Theory, vol. 47, no. 1, pp. 155-172, 2001.

[4] F. Labeau, L. Vandendorpe, and B. Macq, "Structures, factorizations, and design criteria for oversampled paraunitary filterbanks yielding linear-phase filters," IEEE Transactions on Signal Processing, vol. 48, no. 11, pp. 3062-3071, 2000.

[5] T. Tanaka and Y. Yamashita, "On perfect reconstruction with lost channel data in lapped pseudo-orthogonal transform," in Proceedings of 12th European Signal Processing Conference (EUSIPCO '04), vol. 1, pp. 877-880, Vienna, Austria, September 2004.
[6] F. Labeau, L. Vandendorpe, and B. Macq, "Oversampled filter banks as error correcting codes," in Proceedings of 5th International Symposium on Wireless Personal Multimedia Communications (WPMC '02), vol. 3, pp. 1265-1269, Honolulu, Hawaii, USA, October 2002.

[7] F. Labeau, "Design and implementation issues in oversampled filter banks," in Proceedings of 36th Asilomar Conference on Signals, Systems, and Computers, vol. 1, pp. 328-332, Pacific Grove, Calif, USA, November 2002.

[8] F. Labeau, J. C. Chiang, M. Kieffer, P. Duhamel, L. Vandendorpe, and B. Macq, "Oversampled filter banks as error correcting codes: theory and impulse noise correction," IEEE Transactions on Signal Processing, vol. 53, no. 12, pp. 46194630, 2005.

[9] J. Kliewer and A. Mertins, "Error-resilient transmission of waveform signals using overcomplete expansions and softinput source decoding," in Proceedings of 12th European Signal Processing Conference (EUSIPCO '04), vol. 1, pp. 879-882, Vienna, Austria, September 2004.

[10] S. Weiss, "On the design of oversampled filter banks for channel coding," in Proceedings of 12th European Signal Processing Conference (EUSIPCO '04), vol. 1, pp. 885-888, Vienna, Austria, September 2004.

[11] J. G. McWhirter and P. D. Baxter, "A novel technique for broadband singular value decomposition," in Proceedings of 12th Annual Workshop on Adaptive Sensor Array Processing (ASAP '04), MIT Lincoln Laboratory, Lexington, Mass, USA, March 2004.

[12] W. Kellermann, "Analysis and design of multirate systems for cancellation of acoustical echoes," in Proceedings of IEEE International Conference on Acoustics, Speech, and Signal Processing (ICASSP '88), vol. 5, pp. 2570-2573, New York, NY, USA, April 1988.

[13] F. Lorenzelli, A. Wang, D. Korompis, R. Hudson, and K. Yao, "Subband processing for broadband microphone arrays," The Journal of VLSI Signal Processing-Systems for Signal, Image, and Video Technology, vol. 14, no. 1, pp. 43-55, 1996.

[14] S. Weiss, R. W. Stewart, M. Schabert, I. K. Proudler, and M. W. Hoffman, "An efficient scheme for broadband adaptive beamforming," in Proceedings of 33rd Asilomar Conference on Signals, Systems, and Computers, vol. 1, pp. 496-500, Pacific Grove, Calif, USA, October 1999.

[15] W. H. Neo and B. Farhang-Boroujeny, "Robust microphone arrays using subband adaptive filters," IEE ProceedingsVision, Image and Signal Processing, vol. 149, no. 1, pp. 17-25, 2002.

[16] S. Weiss, S. R. Dooley, R. W. Stewart, and A. K. Nandi, "Adaptive equalisation in oversampled subbands," IEE Electronics Letters, vol. 34, no. 15, pp. 1452-1453, 1998.

[17] P. P. Vaidyanathan, Multirate Systems and Filter Banks, Prentice-Hall, Englewood Cliffs, NJ, USA, 1993.

[18] M. Harteneck, S. Weiss, and R. W. Stewart, "Design of near perfect reconstruction oversampled filter banks for subband adaptive filters," IEEE Transactions on Circuits and SystemsPart II: Analog and Digital Signal Processing, vol. 46, no. 8, pp. 1081-1085, 1999.

[19] P. P. Vaidyanathan, "Theory of optimal orthonormal subband coders," IEEE Transactions on Signal Processing, vol. 46, no. 6, pp. 1528-1543, 1998.

[20] S. Redif and T. Cooper, "Paraunitary filter bank design via a polynomial singular-value decomposition," in Proceedings of IEEE International Conference on Acoustics, Speech, and Signal Processing (ICASSP '05), vol. 4, pp. 613-616, Philadelphia, Pa, USA, March 2005. 
[21] A. Papoulis, Probability, Random Variables, and Stochastic Processes, McGraw-Hill, New York, NY, USA, 3rd edition, 1991.

[22] T. Esmailian, F. R. Kschischang, and P. G. Gulak, "In-building power lines as high-speed communication channels: channel characterization and a test channel ensemble," International Journal of Communication Systems, vol. 16, no. 5, pp. 381-400, 2003.

[23] C. Liu, S. Weiss, S. Redif, T. Cooper, L. Lampe, and J. G. McWhirter, "Channel coding for power line communication based on oversampled filter banks," in Proceedings of 9th International Symposium on Power-Line Communications and Its Applications (ISPLC '05), pp. 246-249, Vancouver, British Columbia, Canada, April 2005.

Stephan Weiss received the Dipl.-Ing. degree from the University of ErlangenNürnberg, Erlangen, Germany, in 1995, and the Ph.D. degree from the University of Strathclyde, Glasgow, Scotland, in 1998, both in electronic and electrical engineering. In 1999, he joined the Communications Research Group within the School of Electronics and Computer Science at the University of Southampton, where he is a Se-

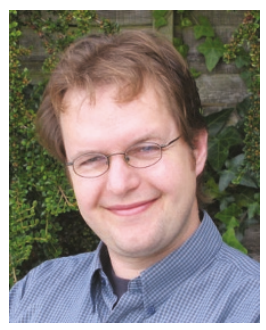
nior Lecturer. Prior to this appointment, he held a Visiting Lectureship at the University of Strathclyde. In 1996/1997, he was a Visiting Scholar at the University of Southern California. His research interests are mainly in adaptive and array signal processing, multirate systems, and signal expansions, with applications in communications, audio, and biomedical signal processing. For his work in biomedical signal processing, he was a corecipient of the $2001 \mathrm{Re}-$ search Award of the German society on hearing aids. He is a Member of the VDE and EURASIP, a Senior Member of the IEEE, and a Member of the IEE Signal Processing Professional Network Executive Team.

Soydan Redif received a B.Eng. (honours) degree in electronic engineering from Middlesex University, London, in 1998. From 1999 to 2000, he was with the Communications Department at the Defence Evaluation and Research Agency, Defford, where he worked on airborne mobile SHF and EHF satellite communications systems. In October 2000, he joined the Advanced Signal and Information Processing Group at

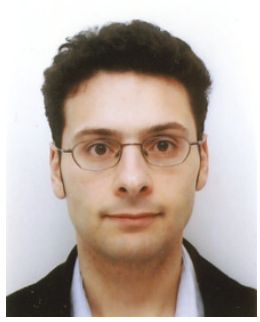
QinetiQ, Malvern, as a Research Scientist. His research contributions have been in adaptive filtering, broadband blind signal separation, multirate systems, and algorithms for polynomial matrix computations. Since October 2002, he has been pursuing a Ph.D. degree at the University of Southampton. He was the recipient of the IEE Award for the best engineering student in 1998. He is a Member of the IEE and a Chartered Engineer.

Tom Cooper received a First-Class B.S. (honours) degree in pure mathematics from Reading University, in 1989. In 1991, he received an M.S. degree, and in 1994 a Ph.D. degree, both in mathematics and both from Warwick University. The subject of his Ph.D. was singularity theory. In 1995, he joined the Defence Research Agency as a Research Scientist, working initially on ocean

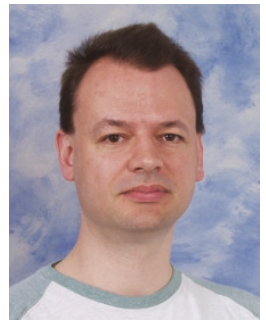

waves. In 2001, he joined QinetiQ's Advanced Signal and Information Processing Group, and has worked on Cramer-Rao bounds and algorithms for direction-of-arrival estimation, polynomial matrix algorithms for signal processing, and algorithms for processing radar data.

Chunguang Liu received the B.Eng. degree in electronics and information engineering from Dalian University of Technology, China, in 2003, and the M.S. degree with distinction in communications systems from the University of Southampton, UK, in 2004. Since October 2004, he has been with the Communications Research Group, in the School of Electronics and Computer Science at the University of Southampton, pursuing postgraduate research in the area of broadband communications systems, specifically the application of filter banks to channel coding and equalisation.

Paul D. Baxter studied mathematics at the University of Cambridge, UK, receiving the B.A. degree in 1998 and completing the Certificate of Advanced Study in mathematics with distinction in 1999. Since then, he has worked in the Advanced Signal and Information Processing Group of QinetiQ, researching in the fields of blind signal separation and convolutive algorithms. He obtained his Ph.D. degree in electrical engi-

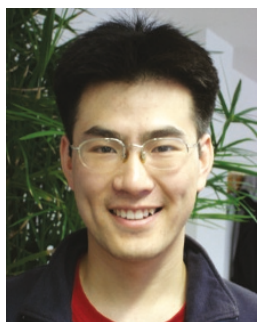
neering from Imperial College, University of London, in 2005, for a thesis entitled "Blind signal separation of convolutive mixtures." He is a Member of the Institute of Mathematics and its Applications and a Chartered Mathematician.

John G. McWhirter gained a First-Class Honours degree in mathematics (1970) and a Ph.D. degree in theoretical physics (1973) from the Queen's University of Belfast. He is currently a Senior Fellow in the Advanced Signal Processing Group at QinetiQ, Malvern. He is also a Visiting Professor in Electrical Engineering at the Queen's University of Belfast and at Cardiff University. He has been carrying out research on adap-

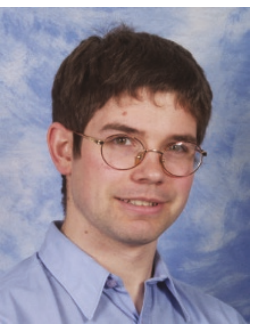
tive signal processing since 1980 and was awarded the J J Thomson Medal by the Institution of Electrical Engineers in 1994 for his research on systolic arrays. He has published more than 130 research papers and holds numerous patents. He was elected as a Fellow of the Royal Academy of Engineering in 1996 and the Royal Society in 1999. He served as President of the Institute of Mathematics and its Applications (IMA) in 2002 and 2003.

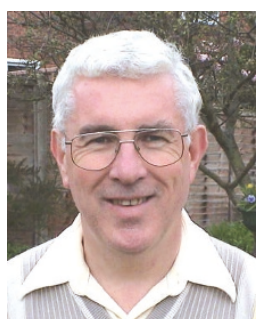

